\title{
CDISC SDTM ARC Coronary Stent Thrombosis Timing Terminology
}

National Cancer Institute

\section{Source}

National Cancer Institute. CDISC SDTM ARC Coronary Stent Thrombosis Timing

Terminology. NCI Thesaurus. Code C119019.

Terminology associated with the ARC coronary stent thrombosis timing responses codelist of the Clinical Data Interchange Standards Consortium (CDISC) Study Data Tabulation Model (SDT M). 